\title{
Distributed Estimation and Control for Large Population Stochastic Multi-Agent Systems with Coupling in the Measurements
}

\author{
Mehdi Abedinpour Fallah *, Roland P. Malhamé * and Francesco Martinelli ${ }^{\dagger}$ \\ * GERAD and Department of Electrical Engineering, \\ École Polytechnique de Montréal, Montreal, Quebec, Canada \\ $\dagger$ Dipartimento di Ingegneria Civile e Ingegneria Informatica, \\ Università di Roma "Tor Vergata", via del Politecnico, I-00133, Rome, Italy \\ Emails: \{mehdi.abedinpour-fallah,roland.malhame\}@polymtl.ca, martinelli@disp.uniroma2.it
}

\begin{abstract}
In this paper, we investigate a class of large population stochastic multi-agent systems where the agents have linear stochastic dynamics and are coupled via their measurement equations. Using the state aggregation technique, we propose a distributed estimation and control algorithm that combines the Kalman filtering for state estimation and the linear-quadratic-Gaussian (LQG) feedback controller. Moreover, the stability analysis in terms of exponential boundedness in the mean square is given for the proposed algorithm.
\end{abstract}

\section{INTRODUCTION}

In recent years, analysis and control design for large population stochastic multi-agent systems have become an active area in the study and control of complex systems [1][6]. Many practical applications and examples of these systems arise in engineering, biological, social and economic fields [7]- [11].

In conventional control systems, control laws are constructed based upon the overall states of the plants. However, in complex systems with many agents, each agent has a self-governed but limited capability of sensing, decisionmaking and communication. Therefore an important issue is the development of decentralized solutions so that each individual agent may implement a strategy based on its local information together with statistical information on the population of agents. Just as stabilization and optimization are two fundamental issues for single-agent systems, for large population stochastic multi-agent systems we are also concerned with how to construct decentralized control laws that preserve closed-loop system stability while optimizing the performance of agents in a cooperative or non-cooperative (the focus of this paper) context.

Agent to agent interaction during competitive decisionmaking is usually due to the coupling in their dynamics or cost functions. Specifically, the dynamic coupling is used to specify an environment effect to the individual's decisionmaking generated by the population of other agents. While each agent only receives a negligible influence from any other given individual, the overall effect of the population (i.e., the mass effect) is significant for each agent's strategy selection.

The state estimation problem has been a fundamental and a challenging problem in theory and applications of control systems. A new formulation of particle filters inspired by the mean-field game theoretic framework of [3], was presented in [12], [13]. Mean field based distributed multiagent decision-making with partial observation was studied in [1], where the considered agents were weakly coupled through both individual dynamics and costs. In this paper, we study a somewhat dual situation whereby large populations of partially observed stochastic agents, although a priori individually independent, are coupled only via their observation structure. More specifically, the "quality" of individual state measurements is affected by certain statistics of the rest of agent states, such as mean, variance, and in the most general case, the instantaneous empirical distribution of these states. It is the latter which in the limit of an infinite population is referred to as the mean field.

Individual agent dynamics are assumed to be linear, stochastic, with linear local state measurements, and in the current paper, the measurements interaction model is assumed to depend only on the empirical mean of agents states, either in a purely additive manner or through the variance of the local measurement. Each agent is associated with an exponentially discounted individual quadratic cost function, and we look for possible, mean field based, Nash equilibrium inducing decentralized control laws as the number of agents grows without bounds.

The study of such measurement-coupled systems is inspired by a variety of applications, for instance the communications model for power control in cellular telephone systems [14], where the received signal of a given user at the base station views all other incell user signals, as well as other cell signals arriving at the base station, as interference or noise. In general, the model is aimed at capturing various forms of interference effects from the environment on individual agent observations.

\section{SYSTEM MODEL AND PROBLEM STATEMENT}

Consider a system of $n$ agents, each with scalar dynamics where the evolution of the state component is described by

$$
d z_{i}=\left(a z_{i}+b u_{i}\right) d t+\sigma_{w} d w_{i}
$$

and the evolution of the measured output is given by either of the following 
- Case (a):

$$
d y_{i}=c z_{i} d t+\left(\sigma_{v}+h\left(\frac{1}{n} \sum_{j=1}^{n} z_{j}\right)\right) d v_{i}
$$

- Case (b):

$$
d y_{i}=\left(c z_{i}+h\left(\frac{1}{n} \sum_{j=1}^{n} z_{j}\right)\right) d t+\sigma_{v} d v_{i}
$$

for $t \geq 0$ and $1 \leq i \leq n$, where $z_{i}(t), u_{i}(t), y_{i}(t) \in \mathbb{R}$ are the state, the control input and the measured output of the $i^{\text {th }}$ agent, respectively. $\left\{w_{i}, v_{i}, 1 \leq i \leq n\right\}$ denotes a sequence of $2 n$ mutually independent standard scalar Wiener processes. The Gaussian initial conditions $z_{i}(0)$ are mutually independent and are also independent of $\left\{w_{i}, v_{i}, 1 \leq i \leq n\right\}$. Moreover, $\sigma_{v}$ is a positive scalar number, and $b, c, h>0$.

The problem to be considered is stated as follows.

Problem1: Design coupled distributed estimation and control strategies based on a feedback control of the form

$$
u_{i}(t)=-f \hat{z}_{i}(t)
$$

where $f>0$ and $\hat{z}_{i}(t)$ is an estimate of $z_{i}(t)$, such that each agent's individual cost function given by

$$
J_{i}\left(u_{i}\right) \triangleq \mathbb{E} \int_{0}^{\infty} e^{-\rho t}\left(z_{i}^{2}+r u_{i}^{2}\right) d t
$$

is optimized utilizing only its local information. Here it is assumed that $\rho, r>0$.

\section{COUPLED DISTRIBUTED ESTIMATION AND CONTROL ALGORITHM}

We combine the Kalman filtering for state estimation and the LQG feedback controller into a closed-loop dynamics model. Noting the information constraints for the agents, the Kalman filtering cannot be directly applied to the $n$ dimensional system. That is also because in our model there is not a central optimizer which can access all other agent's outputs and then form the optimal estimate of the state vector. However, for large $n$, as in the typical mean field analysis [3], we shall assume in the first place that conditions are satisfied so that controlled agents become asymptotically independent (in large population limit), and furthermore the coupling term (mass effect) described by

$$
m(t)=h\left(\frac{1}{n} \sum_{j=1}^{n} z_{j}(t)\right)
$$

for $t \geq 0$ and $1 \leq i \leq n$, is approximated by a deterministic continuous function $m^{*}(t)$ defined on $[0, \infty)$ (to be determined later). It is implicitly predicated on the assumption that the coupling in a stochastic process sense, between agent states becomes sufficiently weak as $n$ grows without bounds, and furthermore, that the individual state variance under state estimate feedback remains bounded, so that by the law of large numbers the average in (6) converges pointwise a.e. to its (deterministic) mean. This leads to uncoupled measurement equations, and therefore the optimal state estimation for $z_{i}$ would be given by the standard scalar Kalman filtering. Now in the large but finite population condition, it is expected that the Kalman filtering structure will still produce a satisfactory estimate when $m(t)$ appears in the measurement equations (2) and (3) but is approximated by $m^{*}(t)$ when constructing the filtering equation. Here we simply proceed by presuming $m^{*}(t)$ as a given deterministic function, and the detailed procedure for obtaining this function will be given after the control synthesis is described. In addition, we establish sufficient conditions under which the variance of $z_{i}$ 's remains indeed bounded. This justifies, after the fact, our initial deterministic assumption.

\section{A. LQG Feedback Controller}

Consider only the dynamic model (1) (without measurement equation (2) or (3)) and assume for the moment that the state $z_{i}$ is completely observable. For minimization of $J_{i}$ defined by (5), the admissible control set is taken as $\mathcal{U}_{i}=\left\{u_{i} \mid u_{i}\right.$ is adapted to the $\sigma$-algebra $\sigma\left(z_{i}(s), s \leq t\right)$, and $\left.J_{i}\left(u_{i}\right)<\infty\right\}$. The set $\mathcal{U}_{i}$ is nonempty due to controllability of (1). Let $f>0$ be the solution to the algebraic Riccati equation

$$
b f^{2}+(\rho-2 a) f-\frac{b}{r}=0
$$

Moreover, if one assumes that $\mathbb{E}\left|z_{i}(0)\right|^{2}<\infty$ and $\beta_{1}=$ $-a+b f>0$, then the control law

$$
u_{i}(t)=-f z_{i}(t)
$$

is stabilizing and further minimizes $J_{i}\left(u_{i}\right)$ for all $u_{i} \in$ $\mathcal{U}_{i}[15]$.

Assumption 1:

$$
a-b f<0
$$

\section{B. Kalman Filter}

Now assume that $z_{i}$ 's are only partially observable and consider either of the two measurement equations (2) or (3). Approximating and replacing $m(t)$ with the same assumed deterministic function $m^{*}(t)$ for all agents, the standard (time-varying) Kalman filter would produce the optimal state estimate using the following algorithm [15]- [16],

$$
\begin{aligned}
d \hat{z}_{i} & =\left(a \hat{z}_{i}+b u_{i}\right) d t+K(t)\left(d y_{i}-c \hat{z}_{i} d t\right) \\
\frac{d P(t)}{d t} & =2 a P(t)+Q-c^{2} R^{-1}(t) P^{2}(t) \\
K(t) & =P(t) c R^{-1}(t)
\end{aligned}
$$

where the process noise covariance matrice is $Q=\sigma_{w}^{2}$ and the measurement noise covariance matrices are chosen as $R_{a}(t)=\left(\sigma_{v}+m^{*}(t)\right)^{2}$ and $R_{b}(t)=\sigma_{v}^{2}$ for case (a) and case (b), respectively. Additionally, the separation principle holding, feedback of the state estimate in (8) would also produce optimal performance.

Assumption 2: All agents have mutually independent Gaussian initial conditions with $\mathbb{E} z_{i}(0)=\zeta_{1}$ and $\mathbb{E} z_{i}^{2}(0)=$ $\zeta_{2}>0$ for all $i$, where $\zeta_{2}>\zeta_{1}^{2}$. 
Remark 1: For agent $i$, the initial condition of the Riccati equation (11) is $\operatorname{Var}\left(z_{i}(0)\right)$ which yields the corresponding solution $P_{i}(t)$. Under Assumption 2, $\operatorname{Var}\left(z_{i}(0)\right)=\zeta_{2}-$ $\zeta_{1}{ }^{2}=\zeta>0$ for all $i$, and therefore the same solution $P(t)$ is obtained for all agents.

\section{State Aggregation}

Assume $m^{*}(t) \in C_{b}[0, \infty)$ is given, where $C_{b}[0, \infty)$ denotes the set of deterministic, bounded and continuous functions on $[0, \infty)$. For the $i^{\text {th }}$ agent, after applying the optimal control law (4), the closed loop equation is

$$
d z_{i}=\left(a z_{i}-b f \hat{z}_{i}\right) d t+\sigma_{w} d w_{i}
$$

Denoting $\bar{z}_{i}(t)=\mathbb{E} z_{i}(t)$ and taking expectation on both sides of (13) gives

$$
\frac{d \bar{z}_{i}}{d t}=a \bar{z}_{i}-b f \mathbb{E} \hat{z}_{i}
$$

with the initial condition $\left.\bar{z}_{i}\right|_{t=0}=\mathbb{E} z_{i}(0)$ assumed and shared by all agents. Also note that in the view of the unbiasedness of the Kalman filter estimate, $\mathbb{E} \hat{z}_{i}=\mathbb{E} z_{i}$. Moreover, the population average of means is defined as $\bar{z} \triangleq(1 / n) \sum_{i=1}^{n} \bar{z}_{i}$ and is simply called the population mean. If as assumed $\bar{z}$ becomes deterministic as $n$ goes to infinity, then because of independence of the individual controlled $z_{i}$ 's under Assumption (2), $\bar{z}$ must converge pointwise to its expectation, i.e. to $\mathbb{E} \bar{z}_{i}$ of a generic agent with initial mean $\zeta_{1}$, where for the considered uniform population of agents:

$$
\frac{d \bar{z}}{d t}=(a-b f) \bar{z}
$$

which yields

$$
\bar{z}(t)=\bar{z}(0) e^{(a-b f) t}
$$

with condition (9) from Assumption 1 guaranteeing boundedness of $\bar{z}(t)$. Here, for simplicity of the analysis, we assume that $\bar{z}(0) \geq 0$.

Furthermore, the population effect $(1 / n) \sum_{j=1}^{n} z_{j}$ is approximated by $\bar{z}$. Since we wish to have

$$
m^{*}(t) \approx m(t)=h\left(\frac{1}{n} \sum_{j=1}^{n} z_{j}(t)\right)
$$

for large $n, m^{*}(t)$ is expressed in terms of the population mean $\bar{z}(t)$ as

$$
m^{*}(t)=h \bar{z}(t)
$$

Remark 2: Under Assumptions 1 and 2, $m^{*}(t)$ does indeed belong to $C_{b}[0, \infty)$.

Remark 3: The state aggregation equation (15) also holds in the case of perfect observation; that is, it is not affected by the partial observation situation.

\section{Proposed Algorithm and Closed-loop Dynamics}

The coupled distributed estimation and control strategies are presented in Algorithm 1. We proceed by obtaining the resultant closed-loop dynamics of the $i^{\text {th }}$ agent and of the population mean.

\section{Solution Algorithm 1}

- Initialization

$$
\hat{z}_{i}(0)=\mathbb{E} z_{i}(0)=\bar{z}(0) \geq 0, P(0)=\zeta>0,1 \leq i \leq n
$$

- State Aggregation

$$
\begin{aligned}
\bar{z}(t) & =\bar{z}(0) e^{(a-b f) t} \\
m^{*}(t) & =h \bar{z}(t)
\end{aligned}
$$

- LQG Feedback Controller

$$
\begin{aligned}
\rho f & =2 a f-b f^{2}+\frac{b}{r} \\
u_{i}(t) & =-f \hat{z}_{i}(t), \quad 1 \leq i \leq n
\end{aligned}
$$

- Kalman filtering

- Case (a):

$$
\begin{aligned}
d \hat{z}_{i} & =\left(a \hat{z}_{i}+b u_{i}\right) d t+K(t)\left(d y_{i}-c \hat{z}_{i} d t\right) \\
\frac{d P(t)}{d t} & =2 a P(t)+\sigma_{w}^{2}-c^{2}\left(\sigma_{v}+m^{*}\right)^{-2} P^{2}(t) \\
K(t) & =P(t) c\left(\sigma_{v}+m^{*}\right)^{-2}
\end{aligned}
$$

- Case (b):

$$
\begin{aligned}
& d \hat{z}_{i}=\left(a \hat{z}_{i}+b u_{i}\right) d t+K(t)\left(d y_{i}-\left(c \hat{z}_{i}+m^{*}\right) d t\right) \\
& \frac{d P(t)}{d t}=2 a P(t)+\sigma_{w}^{2}-c^{2} \sigma_{v}^{-2} P^{2}(t) \\
& K(t)=P(t) c \sigma_{v}^{-2}
\end{aligned}
$$

1) Case (a): Defining the estimation error as

$$
\tilde{z}_{i}=z_{i}-\hat{z}_{i}
$$

and replacing (2) and (4) in (10), yields

$$
\begin{aligned}
d \hat{z}_{i}= & (a-b f) \hat{z}_{i} d t+P(t) c^{2}\left(\sigma_{v}+m^{*}\right)^{-2} \tilde{z}_{i} d t \\
& +P(t) c\left(\sigma_{v}+m^{*}\right)^{-2}\left(\sigma_{v}+h\left(\frac{1}{n} \sum_{j=1}^{n} z_{j}\right)\right) d v_{i}
\end{aligned}
$$

Subsequently, using (1), (19) and (20) it follows that

$$
d z_{i}=(a-b f) z_{i} d t+b f \tilde{z}_{i} d t+\sigma_{w} d w_{i}
$$

and

$$
\begin{aligned}
d \tilde{z}_{i}= & \left(a-P(t) c^{2}\left(\sigma_{v}+m^{*}\right)^{-2}\right) \tilde{z}_{i} d t+\sigma_{w} d w_{i} \\
& -P(t) c\left(\sigma_{v}+m^{*}\right)^{-2}\left(\sigma_{v}+h\left(\frac{1}{n} \sum_{j=1}^{n} z_{j}\right)\right) d v_{i}
\end{aligned}
$$

In addition, letting $z_{n}^{\prime}=(1 / n) \sum_{i=1}^{n} z_{i}, \quad \hat{z}_{n}^{\prime}=$ $(1 / n) \sum_{i=1}^{n} \hat{z}_{i}, \quad \tilde{z}_{n}^{\prime}=(1 / n) \sum_{i=1}^{n} \tilde{z}_{i}, \quad w^{\prime}=$ $(1 / \sqrt{n}) \sum_{i=1}^{n} w_{i}$, and $v^{\prime}=(1 / \sqrt{n}) \sum_{i=1}^{n} v_{i}$, where $w^{\prime}$ and $v^{\prime}$ are two independent standard Wiener processes, we get

$$
d z_{n}^{\prime}=(a-b f) z_{n}^{\prime} d t+b f \tilde{z}_{n}^{\prime} d t+\frac{1}{\sqrt{n}} \sigma_{w} d w^{\prime}
$$


and

$$
\begin{aligned}
d \tilde{z}_{n}^{\prime}= & \left(a-P(t) c^{2}\left(\sigma_{v}+m^{*}\right)^{-2}\right) \tilde{z}_{n}^{\prime} d t+\frac{1}{\sqrt{n}} \sigma_{w} d w^{\prime} \\
& -\frac{1}{\sqrt{n}} P(t) c\left(\sigma_{v}+m^{*}\right)^{-2}\left(\sigma_{v}+h z_{n}^{\prime}\right) d v^{\prime}
\end{aligned}
$$

2) Case (b): Similarly, using (3) in place of (2), it follows that

$$
d z_{i}=(a-b f) z_{i} d t+b f \tilde{z}_{i} d t+\sigma_{w} d w_{i},
$$

$$
\begin{aligned}
d \tilde{z}_{i}= & \left(a-P(t) c^{2} \sigma_{v}^{-2}\right) \tilde{z}_{i} d t-P(t) c \sigma_{v}^{-2} h\left(\frac{1}{n} \sum_{j=1}^{n} z_{j}\right) d t \\
& +P(t) c \sigma_{v}^{-2} m^{*}(t) d t+\sigma_{w} d w_{i}-P(t) c \sigma_{v}^{-1} d v_{i}
\end{aligned}
$$

and

$$
\begin{aligned}
& d z_{n}^{\prime}=(a-b f) z_{n}^{\prime} d t+b f \tilde{z}_{n}^{\prime} d t+\frac{1}{\sqrt{n}} \sigma_{w} d w^{\prime} \\
d \tilde{z}_{n}^{\prime}= & \left(a-P(t) c^{2} \sigma_{v}^{-2}\right) \tilde{z}_{n}^{\prime} d t-P(t) c \sigma_{v}^{-2} h z_{n}^{\prime} d t \\
& +P(t) c \sigma_{v}^{-2} m^{*}(t) d t+\frac{1}{\sqrt{n}} \sigma_{w} d w^{\prime}-\frac{1}{\sqrt{n}} P(t) c \sigma_{v}^{-1} d v^{\prime}
\end{aligned}
$$

\section{STABILITY ANALYSIS}

For stability analysis of the closed-loop dynamics (23)(24) and (27)-(28) we make use of the following concepts of boundedness for solutions of stochastic differential equations.

Definition 1: [17] The stochastic process $x(t)$ is said to be stochastically sample path bounded, if for every $\delta>0$ there is a $\beta(\delta)>0$ such that

$$
\mathbb{P}\left[\sup _{t>0}\|x(t)\| \leq \beta(\delta)\right] \geq 1-\delta
$$

Definition 2: [17] The stochastic process $x(t)$ is said to be exponentially bounded in mean square, if there are real numbers $\eta, \nu, \varphi>0$ such that

$$
\mathbb{E}\|x(t)\|^{2} \leq \varphi\|x(0)\|^{2} \exp (-\eta t)+\nu
$$

holds for every $t \geq 0$.

Definition 3: [17], [18] Consider the continuous-time stochastic process described by the Itô stochastic differential equation,

$$
d x(t)=F(x(t), t) d t+G(x(t), t) d \bar{w}(t)
$$

where $x(t) \in \mathbb{R}^{n_{x}}$ is the state, and $\bar{w}(t) \in \mathbb{R}^{n_{w}}$ is a standard Wiener process. Moreover, the nonlinear functions $F$ and $G$ are assumed to be continuously differentiable, and such that (31) has a unique solution. Consider a nonnegative function $V(x(t), t)$ which is continuously twice differentiable in $x$ and once in $t$, i.e., $V(x, t) \in C^{2,1}$. The differential generator of (31) associated with the Lyapunov function $V(x, t)$ is defined by

$$
\begin{aligned}
\mathcal{L} V(x, t)= & \frac{\partial V}{\partial t}(x, t)+\frac{\partial V}{\partial x}(x, t) F(x, t) \\
& +\frac{1}{2} \sum_{i=1}^{n_{x}} \sum_{j=1}^{n_{x}} \frac{\partial^{2} V}{\partial x_{i} \partial x_{j}}(x, t)\left[G(x, t) G^{T}(x, t)\right]_{i, j}
\end{aligned}
$$

where $x=\left[x_{1}, \ldots, x_{n_{x}}\right]^{T}$, and $\left[G(x, t) G^{T}(x, t)\right]_{i, j}$ is the matrix element of $G(x, t) G^{T}(x, t)$ in the $i^{\text {th }}$ row and the $j^{\text {th }}$ column. Furthermore, the sum in (32) can be simplified as

$$
\begin{aligned}
& \sum_{i=1}^{n_{x}} \sum_{j=1}^{n_{x}} \frac{\partial^{2} V}{\partial x_{i} \partial x_{j}}(x, t)\left[G(x, t) G^{T}(x, t)\right]_{i, j} \\
& =\operatorname{tr}\left(G(x, t) G^{T}(x, t) \operatorname{Hess}_{x}[V(x, t)]\right) \\
& =\operatorname{tr}\left(\operatorname{Hess}_{x}[V(x, t)] G(x, t) G^{T}(x, t)\right)
\end{aligned}
$$

where $\operatorname{Hess}_{x}[\cdot]$ denotes the Hessian matrix with respect to $x$ as the variable vector.

Lemma 1: [17] Assume there is a sufficiently smooth function $V(x(t), t) \in C^{2,1}$ of the stochastic process $x(t)$ in (31) and real numbers $\vartheta_{\min }, \vartheta_{\max }, \mu, \alpha>0$ such that

$$
\vartheta_{\min }\|x(t)\|^{2} \leq V(x(t), t) \leq \vartheta_{\max }\|x(t)\|^{2}
$$

and

$$
\mathcal{L} V(x(t), t) \leq-\alpha V(x(t), t)+\mu
$$

are satisfied. Then the stochastic process $x(t)$ is exponentially bounded in mean square, i.e.,

$$
\mathbb{E}\|x(t)\|^{2} \leq \frac{\vartheta_{\max }}{\vartheta_{\min }}\|x(0)\|^{2} \exp (-\alpha t)+\frac{\mu}{\vartheta_{\min } \alpha}
$$

for every $t \geq 0$. Moreover, the stochastic process is samplepath bounded.

Lemma 2: Each system described by scalar dynamics (1) and either of

$$
d y_{i}=c z_{i} d t+\left(\sigma_{v}+m^{*}(t)\right) d v_{i}
$$

or

$$
d y_{i}=\left(c z_{i}+m^{*}(t)\right) d t+\sigma_{v} d v_{i}
$$

is uniformly detectable.

Proof: For $c>0$, any real number $\Lambda$ such that $\Lambda<\frac{-a}{c}$ yields $a+\Lambda c<0$. Therefore, according to definition 4.1 in [17], the system is uniformly detectable.

Lemma 3: [17] For each uniformly detectable system described by (1) and either of (37) or (38), there are real numbers $p_{\min }, p_{\max }>0$ such that the solution $P(t)$ of the scalar Riccati differential equation (11) satisfies the bounds

$$
p_{\min } \leq P(t) \leq p_{\max }
$$

for every $t \geq 0$.

Theorem 1: If

$$
f>\frac{2 a}{b}
$$

and also if there exists a fixed real number $l$ such that

$$
\frac{2 p_{\max } c^{2} h^{2}}{n \sigma_{v}^{4}(b f-2 a)}<l<\frac{\sigma_{w}^{2}}{b f p_{\max }^{2}}+\frac{c^{2}}{b f\left(\sigma_{v}+h \bar{z}(0)\right)^{2}},
$$

then the stochastic process $x(t)=\left[\begin{array}{c}z_{n}^{\prime}(t) \\ \tilde{z}_{n}^{\prime}(t)\end{array}\right]$ verifying the closed-loop dynamics (23)-(24) for $1 \leq i \leq n$, is exponentially bounded in mean square and stochastically sample-path 
bounded. Specifically,

$$
\begin{aligned}
\mathbb{E}\left(z_{n}^{\prime 2}(t)+\tilde{z}_{n}^{\prime 2}(t)\right) \leq & \frac{\max \left\{l, \frac{1}{p_{\min }}\right\}}{\min \left\{l, \frac{1}{p_{\max }}\right\}}\left(z_{n}^{\prime 2}(0)+\tilde{z}_{n}^{\prime 2}(0)\right) e^{-\alpha t} \\
& +\frac{\left(l+\frac{1}{p_{\min }}\right) \frac{\sigma_{w}^{2}}{n}+\frac{2 p_{\max } c^{2}}{n \sigma_{v}^{2}}}{\min \left\{l, \frac{1}{p_{\max }}\right\} \alpha}
\end{aligned}
$$

holds with

$$
\begin{aligned}
\alpha= & \frac{1}{\max \left\{l, \frac{1}{p_{\min }}\right\}} \min \left\{l(b f-2 a)-\frac{2 p_{\max } c^{2} h^{2}}{n \sigma_{v}^{4}},\right. \\
& \left.-l b f+\frac{\sigma_{w}^{2}}{p_{\max }^{2}}+\frac{c^{2}}{\left(\sigma_{v}+h \bar{z}(0)\right)^{2}}\right\}
\end{aligned}
$$

Proof of Theorem 1: Choosing

$$
V(x(t), t)=x^{T}(t) \Pi(t) x(t)
$$

with

$$
\Pi(t)=\left[\begin{array}{cc}
l & 0 \\
0 & P^{-1}(t)
\end{array}\right]
$$

where $l$ is a fixed real number verifying (41) and applying Lemma (3) we can write

$$
l z_{n}^{\prime 2}+\frac{1}{p_{\max }} \tilde{z}_{n}^{\prime 2} \leq V(x, t) \leq l z_{n}^{2}+\frac{1}{p_{\min }} \tilde{z}_{n}^{\prime 2}
$$

Therefore, (34) is verified with $\vartheta_{\min }=\min \left\{l, \frac{1}{p_{\max }}\right\}$ and $\vartheta_{\max }=\max \left\{l, \frac{1}{p_{\min }}\right\}$.

Next, considering the dynamic equations (23)-(24) as in the form of (31) with

$$
F(x, t)=\left[\begin{array}{c}
(a-b f) z_{n}^{\prime}+b f \tilde{z}_{n}^{\prime} \\
\left(a-P(t) c^{2}\left(\sigma_{v}+m^{*}\right)^{-2}\right) \tilde{z}_{n}^{\prime}
\end{array}\right], \bar{w}(t)=\left[\begin{array}{c}
w^{\prime} \\
v^{\prime}
\end{array}\right]
$$

and

$$
G(x, t)=\left[\begin{array}{cc}
\frac{1}{\sqrt{n}} \sigma_{w} & 0 \\
\frac{1}{\sqrt{n}} \sigma_{w} & -\frac{1}{\sqrt{n}} P(t) c\left(\sigma_{v}+m^{*}\right)^{-2}\left(\sigma_{v}+h z_{n}^{\prime}\right)
\end{array}\right]
$$

and using (32) we have

$$
\begin{aligned}
\mathcal{L} V(x, t)= & x^{T}(t) \frac{d \Pi(t)}{d t} x(t)+2 x^{T}(t) \Pi(t) F(x, t) \\
& +\frac{1}{2} \operatorname{tr}\left[G(x, t) G^{T}(x, t) \operatorname{Hess}_{x}(V(x, t))\right]
\end{aligned}
$$

which can be expressed as

$$
\begin{aligned}
\mathcal{L} V= & \left(2 a P^{-1}(t)-2 c^{2}\left(\sigma_{v}+m^{*}\right)^{-2}-\dot{P}(t) P^{-2}(t)\right) \tilde{z}_{n}^{\prime 2} \\
& +2 l(a-b f) z_{n}^{\prime 2}+2 l b f z_{n}^{\prime} \tilde{z}_{n}^{\prime}+\operatorname{tr}\left[G G^{T} \Pi(t)\right]
\end{aligned}
$$

Expressing $\dot{P}(t)$ by means of Riccati differential equation (11), and applying the inequality $2 z_{n}^{\prime} \tilde{z}_{n}^{\prime} \leq z_{n}^{\prime 2}+\tilde{z}_{n}^{\prime 2}$ yields

$$
\begin{aligned}
\mathcal{L} V(x, t) \leq & l(2 a-b f) z_{n}^{\prime 2}+\left(l b f-\sigma_{w}^{2} P^{-2}(t)\right) \tilde{z}_{n}^{\prime 2} \\
& -c^{2}\left(\sigma_{v}+m^{*}\right)^{-2} \tilde{z}_{n}^{2}+\frac{1}{n}\left(l+P^{-1}(t)\right) \sigma_{w}^{2} \\
& +\frac{1}{n} P(t) c^{2}\left(\sigma_{v}+m^{*}\right)^{-4}\left(\sigma_{v}+h z_{n}^{\prime}\right)^{2}
\end{aligned}
$$

In addition, using the bounds from Lemma 3 , applying the inequality $2 \sigma_{v} h z_{n}^{\prime} \leq \sigma_{v}^{2}+h^{2} z_{n}^{\prime 2}$ and noting that $\min _{t}\left\{\sigma_{v}+\right.$ $\left.m^{*}(t)\right\} \geq \sigma_{v}>0$ and also $\max _{t}\left\{\sigma_{v}+m^{*}(t)\right\} \leq \sigma_{v}+h \bar{z}(0)$, after some simplification in the right-hand side of (51), it can be written as

$$
\begin{aligned}
\mathcal{L} V & \leq\left(l(2 a-b f)+\frac{2 p_{\max } c^{2} h^{2}}{n \sigma_{v}^{4}}\right) z_{n}^{\prime 2}+\left(l+\frac{1}{p_{\min }}\right) \frac{\sigma_{w}^{2}}{n} \\
& +\left(l b f-\sigma_{w}^{2} P^{-2}(t)-c^{2}\left(\sigma_{v}+h \bar{z}(0)\right)^{-2}\right) \tilde{z}_{n}^{\prime 2}+\frac{2 p_{\max } c^{2}}{n \sigma_{v}^{2}}
\end{aligned}
$$

Enforcing conditions (40) and (41) in (52) and using the bounds from Lemma 3, inequality (35) holds with $\alpha$ defined in (43) and

$$
\mu=\left(l+\frac{1}{p_{\min }}\right) \frac{\sigma_{w}^{2}}{n}+\frac{2 p_{\max } c^{2}}{n \sigma_{v}^{2}}
$$

Therefore, it follows that (42) holds according to (36). This ends the proof for case (a).

Theorem 2: If

$$
f>\frac{a}{b},
$$

then the stochastic process $x(t)=\left[\begin{array}{c}z_{n}^{\prime}(t) \\ \tilde{z}_{n}^{\prime}(t)\end{array}\right]$ verifying the closed-loop dynamics (27)-(28) for $1 \leq i \leq n$, is exponentially bounded in mean square and stochastically sample-path bounded. Specifically,

$$
\begin{aligned}
\mathbb{E}\left(z_{n}^{\prime 2}(t)+\tilde{z}_{n}^{\prime 2}(t)\right) & \leq \frac{\max \left\{l, \frac{1}{p_{\min }}\right\}}{\min \left\{l, \frac{1}{p_{\max }}\right\}}\left(z_{n}^{\prime 2}(0)+\tilde{z}_{n}^{\prime 2}(0)\right) e^{-\alpha t} \\
& +\frac{\left(l+\frac{1}{p_{\min }}\right) \frac{\sigma_{w}^{2}}{n}+\frac{p_{\max } c^{2}}{n \sigma_{v}^{2}}+\sigma_{v}^{-2} h^{2} \bar{z}^{2}(0)}{\min \left\{l, \frac{1}{p_{\max }}\right\} \alpha}
\end{aligned}
$$

holds with

$$
\alpha=\frac{1}{\max \left\{l, \frac{1}{p_{\min }}\right\}} \min \left\{2 l(b f-a), \frac{\sigma_{w}^{2}}{p_{\max }^{2}}\right\}
$$

and a fixed real number $l=\frac{c h \sigma_{v}^{-2}}{b f}$.

Proof of Theorem 2: Considering the dynamic equations (27)-(28) in place of equations (23)-(24) and following a similar procedure as in Theorem 1, this theorem can be proved for case (b). The details are omitted here for brevity.

Remark 4: Note that under our stability condition (40), inequality (42) is consistent with our initial assumption that as $n$ goes to infinity $z_{n}^{\prime}(t)$ becomes deterministic. Indeed, it confirms that as $n$ goes to infinity, the variance of $z_{n}^{\prime}(t)$ under measurement model (a), goes to zero, provided the initial mean of $z_{n}^{\prime}$ is known to all agents. Similarly, under stability condition (54), inequality (55) indicates that if the initial agents' mean estimate of $\bar{z}(0)$ is correct and equal to zero, the variance of $z_{n}^{\prime}(t)$ under measurement model (b), goes to zero. The same result can be shown to remain true if the initial mean of $\bar{z}(0)$ is strictly positive, but again correctly estimated by all agents. 


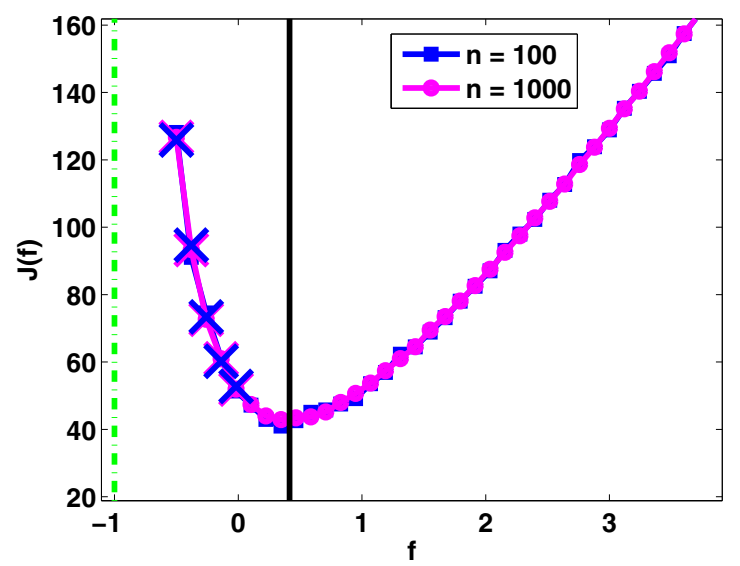

Fig. 1. The LQ cost $J$ as a function of $f$. The black vertical line indicates $f_{L Q G}^{*}$. The dashed green vertical line represents the bound $\frac{2 a}{b}$. The crosses denote the points where one of the two conditions of Theorem 1 is not met.

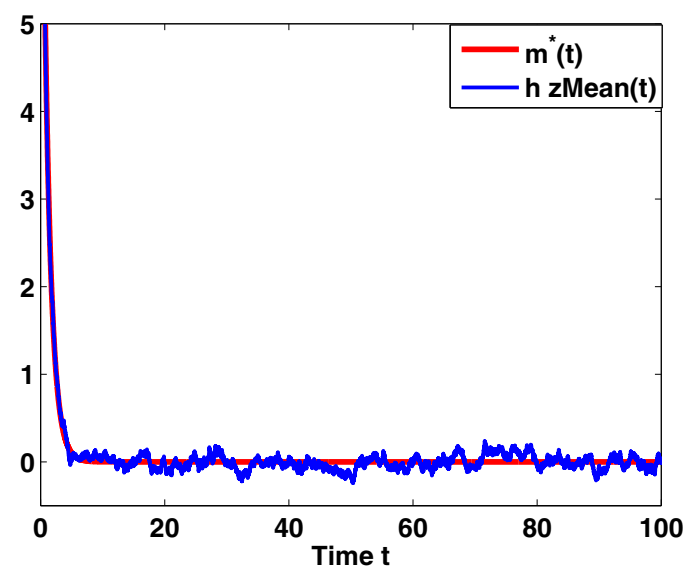

Fig. 2. Comparison between $m^{*}(t)$ and $h\left(\frac{1}{n} \sum_{j=1}^{n} z_{j}(t)\right)$ for $n=100$

\section{NUMERICAL EXAMPLE}

The following numerical values were used with noise model (a): $a=-0.5, b=c=h=\sigma_{w}=\sigma_{v}=$ $\rho=r=1, \mathbb{E} z_{i}(0)=10$ and $\operatorname{Var}\left(z_{i}(0)\right)=1$. The simulation results as depicted in Figures 1 and 2 illustrate a case where $f_{L Q G}^{*}$ satisfies the bound of Theorem 1 , thus guaranteeing convergence of the mean state when $f_{L Q G}^{*}$ is used, to a deterministic value. In this case, $f_{L Q G}^{*}$ induces a Nash equilibrium. It also happens to be a socially optimal equilibrium. However, in general the separation principle will not hold for noise model (a) even as $n$ goes to infinity.

\section{CONCLUSIONS}

This paper addressed the distributed decision-making in a system of uniform agents coupled via their measurement equations, whereby each agent has noisy measurements of its own state. Specifically, a distributed estimation and control algorithm was developed using a decentralized control synthesis in which each agent utilizes an estimate based on its local information and a priori (shared) information on the initial mean state estimate for its control strategy. One special feature of the proposed algorithm is the fact that it combines the Kalman filtering for state estimation and the linearquadratic-Gaussian (LQG) feedback controller based on the anticipation of the collective effect (mean field) of all agents and using the state aggregation technique to anticipate that effect. It was proved that under certain conditions the closedloop dynamics is exponentially bounded in mean square and stochastically sample-path bounded.

\section{ACKNOWLEDGMENTS}

This research was stimulated by a question raised by Professor Prashant G. Mehta at UIUC concerning the possible dualization of mean field control results from control to estimation situations.

\section{REFERENCES}

[1] M. Huang, P.E. Caines, and R.P. Malhamé, "Distributed multi-agent decision-making with partial observations: asymptotic Nash equilibria", Proc. the 17th Internat. Symposium on Math. Theory on Networks and Systems (MTNS'06), Kyoto, Japan, pp. 2725-2730, July 2006.

[2] J.M. Lasry and P.L. Lions, Mean field games, Jpn. J. Math., vol. 2, no. 1, pp. 229260, 2007.

[3] M. Huang, P.E. Caines, and R.P. Malhamé, Large-population costcoupled LQG problems with non-uniform agents: individual-mass behavior and decentralized epsilon-Nash equilibria, IEEE Trans. Autom. Control, vol. 52, no. 9, pp. 1560-1571, Sept. 2007.

[4] T. Li, J. F. Zhang, Asymptotically optimal decentralized control for large population stochastic multi-agent systems, IEEE Trans. Autom. Control, 53(7), pp. 1643-1660, August 2008.

[5] B. C. Wang and J. F. Zhang, Mean Field Games for Large-Population Multiagent Systems with Markov Jump Parameters, SIAM J. Control Optim., vol. 50, no. 4, pp. 2308-2334, 2012.

[6] M. Huang, P.E. Caines, and R.P. Malhamé, Social optima in mean field LQG control: centralized and decentralized strategies, IEEE Trans. Autom. Control, vol. 57, no. 7, pp. 1736-1751, July 2012.

[7] C.Y. Chong, S.P. Kumar, and B.A. Hamilton, Sensor networks: Evolution, opportunities, and challenges, Proc. IEEE, vol. 91, no. 8, pp. 1247-1256, Aug. 2003.

[8] J. H. Reif and H. Y. Wang, Social potential fields: A distributed behavioral control for autonomous robots, Robotics Autonomous Syst., vol. 27, pp. 171-194, 1999.

[9] K.M. Passino, Biomimicry of bacterial foraging: For distributed optimization and control, IEEE Control Syst. Mag., vol. 22, no. 3, pp. 52-67, Jun. 2002.

[10] D. Helbing, I. Farkas, and T. Vicsek, Simulating dynamic features of escape panic, Nature, vol. 407, no. 6803, pp. 487-490, 2000.

[11] A. Lachapelle, Human Crowds and Groups Interactions: A Mean Field Games Approach, Technical report, CEREMADE, Université ParisDauphine, 2010.

[12] T. Yang, P. G. Mehta, and S. P. Meyn, "A mean-field controloriented approach to particle filtering", In Proc. of American Control Conference (ACC2011), San Francisco, CA, USA, June 29-July 01, pp. 2037-2043.

[13] T. Yang, P. G. Mehta, and S. P. Meyn, "Feedback particle filter with mean-field coupling", In Proc. of IEEE Conference on Decision and Control and European Control Conference (CDC-ECC), Orlando, FL, USA, December 2011, pp. 7909-7916.

[14] M. Huang, P.E. Caines, and R.P. Malhamé, Uplink power adjustment in wireless communication systems: a stochastic control analysis, IEEE Trans. Autom. Control, vol. 49, pp. 1693-1708, Oct. 2004.

[15] A. Gelb, Applied Optimal Estimation, MIT Press, Cambridge, MA, 1974.

[16] B. D. O. Anderson and J. B. Moore, Optimal Filtering, Prentice-Hall. Englewood Cliffs. NJ., 1979.

[17] K. Reif, S. Günther, E. Yaz and R. Unbehauen, Stochastic stability of the continuous-time extended Kalman filter, IEE Proc. Control Theory Appl., vol. 147, no. 1, pp. 45-52, Jan. 2000.

[18] X. Mao and C. Yuan, Stochastic Differential Equations with Markovian Switching, Imperial College Press, London, UK, 2006. 